Ann. Génét. Sél. anim., I978, 10 (2), I9I-208.

\title{
Fréquence du syndrome d'hyperthermie maligne dans des populations porcines françaises ; relation avec le développement musculaire
}

\author{
L. OLLIVIER, P. SELLIER et G. MONIN $(*)$
}

avec la collaboration technique de Marie-Reine LANGLors, P. Dando, C. Felgines, A. Talmant $(*)$, D. Tastu et P. Vernin $\left({ }^{*}\right)$

Station de Génétique quantitative et appliquée

Centre national de Recherches zootechniques, I.N.R.A. 78350 Jouy-en-Josas

(*) Station de Recherches sur la viande

Centre de Recherches de Clermont-Ferrand, I.N.R.A. Theix 63 IIO Beaumont

\section{Résumé}

A fin d'estimer la fréquence du syndrome d'hyperthermie maligne (SHM) dans 5 populations, 872 porcs ont été soumis, en $1975^{-1976}$, à une épreuve d'anesthésie à l'halothane, à un poids vif compris entre 20 et $35 \mathrm{~kg}$. Les populations concernées sont : (I) une lignée Piétrain (PA) sélectionnée sur la vitesse de croissance et l'épaisseur de lard dorsal, (2) une lignée Piétrain (PB) sélectionnée en faveur de 1'hypertrophie musculaire, (3) la race Landrace Belge (LBB), (4) la race Landrace Français $(\mathrm{LF}),(5)$ la race Large White $(\mathrm{LW})$. Dans les lignées Piétrain, entretenues dans un troupeau expérimental, tous les porcelets sevrés dans deux générations successives $\left(\mathrm{F}_{2}\right.$ et $\left.\mathrm{F}_{3}\right)$ ont été anesthésiés. Dans les autres races, le test à l'halothane a été réalisé sur des jeunes verrats provenant de plusieurs élevages de sélection et envoyés en station de contrôle individuel. Les effectifs de porcs testés sont $\mathrm{I} 47, \mathrm{I} 88,82, \mathrm{1} 27, \mathrm{I} 28,98$ et 102 , respectivement pour les échantillons $\mathrm{PA}_{2} \mathrm{~F}_{2}, \mathrm{PA}-\mathrm{F}_{3}, \mathrm{~PB}-\mathrm{F}_{2}, \mathrm{~PB}-\mathrm{F}_{3}, \mathrm{LB}, \mathrm{LF}$ et $\mathrm{LW}$. Une note visuelle de développement musculaire a été attribuée à chaque porc au moment de l'épreuve d'anesthésie. Le gain moyen quotidien et l'épaisseur de lard dorsal à $80-85 \mathrm{~kg}$ ont été mesurés sur tous les porcs ainsi que l'indice de consommation chez les porcs LB, LF et LW.

Les pourcentages de sujets positifs ont été respectivement de $27,2 \pm 5,4 ; 34,0 \pm 8,2$; $40,2 \pm 5,9 ; 47,2 \pm 8,3 ; 53, \mathrm{I} \pm 4,4$ et $\mathrm{I}_{8,4} \pm 3,9$ dans les échantillons PA-F2, PA-F $3, \mathrm{~PB}-\mathrm{F}_{2}$, $\mathrm{PB}-\mathrm{F}_{3}, \mathrm{LB}$ et $\mathrm{LF}$; aucune réaction positive $n$ ' a été observée dans l'échantillon $L_{W} W$. La mortalité directement consécutive au test à l'halothane chez les porcs positifs s'est élevée à environ ro p. I oo. Faisant l'hypothèse aujourd'hui bien établie que le SHM est dû à un gène autosomal récessif, nous avons estimé la fréquence de ce gène et sa pénétrance chez l'homozygote : les valeurs trouvées pour les échantillons $\mathrm{PA}-\mathrm{F}_{2}, \mathrm{PA}-\mathrm{F}_{3}, \mathrm{~PB}-\mathrm{F}_{2}, \mathrm{~PB}-\mathrm{F}_{3}, \mathrm{~L}_{1}$ et $\mathrm{LF}_{1}$ sont respectivement $0,57 \pm 0,13$; $0,76 \pm 0,14 ; 0,89 \pm 0,20 ; 0,83 \pm 0,16 ; 0,91 \pm 0,15$ et $0,38 \pm 0,10$ pour la fréquence génique et $0,82 \pm 0,29 ; 0,59 \pm 0,17 ; 0,50 \pm 0,24 ; 0,69 \pm 0,23 ; 0,64 \pm 0,22$ et $\mathrm{x}, 30 \pm 0,65$ pour la pénétrance. La variation entre races de la fréquence du SHM correspond donc à des fréquences différentes du gène responsable et peut-être aussi à une plus ou moins forte pénétrance de ce gène. $\mathrm{L}_{\mathrm{a}}$ fréquence génique est légèrement surestimée chez le $\mathrm{LB}$, du fait de la sélection sur conformation pratiquée par l'éleveur pour le choix des jeunes verrats envoyés en station. 
L'effet du type de réaction à l'halothane sur les caractères de croissance n'est pas significatif mais, quel que soit l'échantillon, la vitesse de croissance tend à être plus faible (environ - I $5 \mathrm{~g} / \mathrm{j}$ ) chez les porcs positifs. L'avantage de ces derniers pour l'épaisseur de lard dorsal est de 0,7 -o,8 mm dans les différents échantillons et est généralement significatif. Pour les 5 populations étudiées, la fréquence du SHM est positivement liée à la note moyenne de développement musculaire; l'avantage des porcs positifs de ce point de vue est significatif dans les échantillons PA, PB et LF mais pas dans l'échantillon $L_{B} B$, pour la raison donnée précédemment. Au total, cette étude montre la liaison génétique étroite entre SHM et développement musculaire; intra-race, cette liaison est particulièrement bien démontrée par la divergence obtenue entre les deux lignées Piétrain pour la fréquence du SHM, comme conséquence de la sélection de l'une d'elles pour l'hypertrophie musculaire. Il subsiste cependant entre races des différences de développement musculaire qui ne peuvent pas être attribuées au locus du syndrome d'hyperthermie maligne.

\section{Introduction}

Le syndrome d'hyperthermie maligne (SHM) est actuellement, chez le Porc, l'objet de nombreuses études dont la quasi-totalité reposent sur l'examen de la réaction de l'animal à l'administration d'un agent anesthésiant, l'halothane. La réponse observée est de type "tout ou rien ": 1'halothane déclenche, chez certains individus, un ensemble de réactions très caractéristiques (HALL et al., I966; Sybesma et EIKELENBoom, I969), qui rappellent étroitement les symptômes accompagnant les cas de mort subite rencontrés dans certaines races porcines. La première étude menée en France sur ce sujet (OLLIVIER et al., I975; Monin et al., I976) a été réalisée dans un troupeau expérimental de porcs Piétrain, race à fort développement musculaire connue pour sa sensibilité particulière au stress. Plusieurs études étrangères sur le test à l'halothane ayant montré que le syndrome d'hyperthermie maligne existe dans d'autres races porcines, nous avons cherché à estimer sa fréquence dans les principales races exploitées en France (Large White, Landrace Français, Landrace Belge, Piétrain). Bien que certains auteurs comme WiLLI,AMs et al. (I975) aient émis une autre hypothèse, il paraît aujourd'hui bien établi, au vu des résultats de Minkema et al. (I977) et de Smith et Bampton (I977), que la sensibilité à l'halothane est due à un gène autosomal récessif, hypothèse d'abord avancée par CHRISTIAN (I972) et en accord avec nos premières observations (OLI IVIER et al., I975). Partant de cette hypothèse, une méthode proposée par LEFORT et al. (I975) permet d'estimer simultanément dans une population la fréquence $d u$ gène responsable $d u S H M$ et sa pénétrance chez les homozygotes. Nous avons appliqué cette méthode aux différentes populations étudiées et nous donnons ici les résultats définitifs de cette analyse; l'estimation des mêmes paramètres avait fait l'objet d'une première analyse partielle dont les résultats ont été présentés par OLLIVIER et al. (I977). Le second objectif de notre étude était de préciser les relations entre le SHM et un certain nombre de caractères mesurables sur l'animal vivant. Nous présentons ici ce qui concerne les caractères de croissance, l'adiposité et le développement musculaire, apprécié visuellement sur le jeune animal. L'étude des liaisons entre le SHM et divers critères sanguins (CPK, LDH, acide lactique, électrolytes) fera l'objet d'un autre article (MoNIN et al., I979). Rappelons aussi qu'une partie des animaux de la présente étude a été utilisée pour la mise en évidence de relations entre le SHM et les réponses osmotiques érythrocytaires (KING et al., I976) et entre le SHM, le taux de créatinine plasmatique et certains polymorphismes enzymatiques (ANSAY et OLLIVIER, I978). 


\section{Matériel et méthodes}

\section{I. - Matériel animal et modalités expérimentales}

Entre février I975 et décembre I976, 872 porcs appartenant à 5 " populations " ont été soumis, à un poids compris entre 20 et $35 \mathrm{~kg}$, à un test d'anesthésie à l'halothane. La technique utilisée s'inspire étroitement de celle décrite par EI KELENBOOM et MIN KEMA (I974). L'anesthésie générale de l'animal est provoquée par inhalation, en circuit fermé, d'un mélange d'oxygène pur (4 litres/minute) et d'halothane (Fluothane ${ }^{\circledast}$, I.C.I. Pharma). La concentration d'halothane est initialement de $4 \mathrm{p}$. Ioo et elle est abaissée à $2,5 \mathrm{p}$. Ioo au début de l'anesthésie effective (perte du clignement réflexe des paupières). A compter de cet instant, l'anesthésie est prolongée 5 minutes exactement en cas d'absence de réaction anormale de l'animal (sujets dits "négatifs "). Chez les animaux qui développent le syndrome (sujets dits " positifs "), l'épreuve est arrêtée dès les premiers symptômes (en particulier raidissement musculaire, surtout visible au niveau des membres postérieurs). La température rectale de l'animal est enregistrée en permanence et ses valeurs initiale (début de l'anesthésie effective) et finale (enlèvement du " masque ") sont notées.

Pour chacun des échantillons, les effectifs de porcs testés, ainsi que le nombre de pères et de mères, sont donnés dans le tableau I. Les données concernant le Piétrain ont été recueillies au domaine expérimental de 1'I.N.R.A., situé à Avord

\section{TABLEAU I}

Effectifs de porcs testés et structure génétique de l'échantillon pour les 5 populations étudiées.

Numbers of pigs, sives and dams in the samples from the 5 populations under study

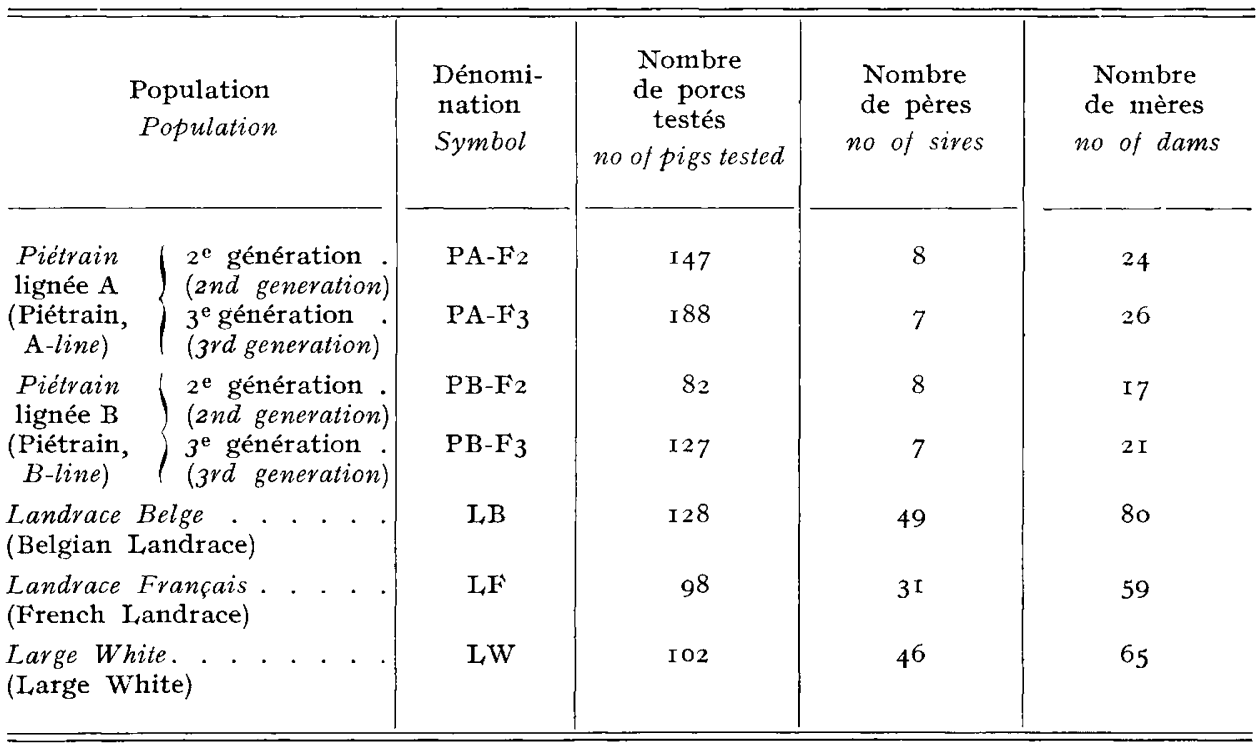


(Cher), dans deux lignées distinctes constituées en I973 à partir d'une fondation commune et sélectionnées depuis lors de façon différente : les animaux testés appartiennent à $1 \mathrm{a} 2^{\mathrm{e}}(\mathrm{F} 2)$ et à $\mathrm{la}^{\mathrm{e}}\left(\mathrm{F}_{3}\right)$ génération de sélection. Dans la lignée $\mathrm{A}$, l'objectif est d'améliorer la vitesse de croissance à l'engraissement et de réduire l'épaisseur du lard dorsal : le critère de sélection est un indice de contrôle individuel combinant ces deux caractères. Dans la lignée $B$, la sélection vise à accroître le degré d'hypertrophie musculaire et le critère de sélection est une note visuelle de développement musculaire donnée, au poids de $20-25 \mathrm{~kg}$, selon la méthode décrite par OIIIVIER et LAUVERGNE (I967); cette note de pointage, comprise entre o et I 2 , est établie en considérant quatre " sites » de manifestation de l'hypertrophie musculaire et en donnant pour chaque site une note de 3,2 , I ou o selon que cette manifestation est très marquée, marquée, atténuée ou absente. Pour les 4 échantillons Piétrain, tous les porcelets présents (mâles et femelles) ont été soumis au test à 1'halothane après avoir été pointés sur conformation. Dans les trois autres populations (Landrace Belge, Landrace Français, Large White), nous avons testé des échantillons de jeunes verrats issus d'élevages de sélection et envoyés en station de contrôle individuel. Les données proviennent de cinq bandes de verrats contrôlés dans deux stations - Gannat (Allier) et Le Transloy (Pas-de-Calais) —, une à trois races étant représentées dans chaque bande. Une note de développement musculaire a été également donnée à ces animaux en utilisant la même méthode que pour le Piétrain.

Tous les animaux ont été contrôlés individuellement. Sur les porcs Piétrain, alimentés ad libitum, les caractères mesurés sont le gain moyen quotidien de 25 à $80 \mathrm{~kg}$ et l'épaisseur de lard dorsal à $80 \mathrm{~kg}$. Pour les animaux des trois autres races, 1'alimentation est de type semi-ad libitum ( 2 repas/jour) et les caractères mesurés sont le gain moyen quotidien et l'indice de consommation entre 35 et $85 \mathrm{~kg}$ et l'épaisseur de lard dorsal à $85 \mathrm{~kg}$. Dans les deux cas, l'épaisseur de lard dorsal est la moyenne de six mesures aux ultra-sons, à raison de 2 mesures prises à $4 \mathrm{~cm}$ de part et d'autre de l'épine dorsale au passage de sangles (" cou »), au niveau de la dernière côte (" dos ") et sur une verticale située à égale distance du grasset et de l'arrière des jambons (" rein ").

\section{2. - Analyse statistique}

A 1'aide de la méthode de LEFORT et al. (I975), nous avons estimé pour chaque échantillon la fréquence $q$ du gène récessif responsable du syndrome, ainsi que sa pénétrance $w$ définie comme la proportion des individus homozygotes pour ce gène qui sont effectivement reconnus positifs lors de l'épreuve d'anesthésie.

Dans le cas des lignées Piétrain, qui ont un effectif limité ( 7 ou 8 pères, 20 à 25 mères), à la variance d'échantillonnage des estimées de $q$ on a ajouté la variance de dérive génétique, soit $\mathrm{F} q(\mathrm{I}-q)$ où $\mathrm{F}$ est 1 'accroissement du coefficient de consanguinité calculé d'après les pedigrees depuis la génération initiale. Cette dérive affecte aussi l'estimation de la proportion $q^{2} w$ de sujets positifs et la variance de dérive de $q^{2} w$ est égale à $w^{2} \mathrm{~V}\left(q^{2}\right)$ si on suppose $w$ d'origine non génétique. Cette expression fait intervenir le moment du $4^{\mathrm{e}}$ ordre de $q$ dont 1'expression en fonction de F et $q$ est donnée par Crow et Kimura (I970, p. 3I9).

Les différences entre sujets positifs et sujets négatifs ont été estimées, pour les caractères énumérés ci-dessus, par la méthode des moindres carrés (HARVEY, r975). Nous avons analysé séparément trois ensembles de données : (r) échantillons PA-F2 et PB-F2; (2) échantillons PA-F3 et PB-F3; (3) échantillons $\mathrm{L}_{1} B, \mathrm{~L}_{1} \mathrm{~F}$ et $\mathrm{LW}$. 
Dans les ensembles (I) et (2), le modèle de base comprenait les facteurs " type de réaction ", "sexe " et " lignée ", ainsi que les trois interactions de rer ordre entre ces facteurs. Dans l'ensemble (3), le modèle de base comprenait les facteurs "type de réaction ", "bande de contrôle " et " race ", ainsi que l'interaction " type de réaction" $x$ " race ". D'autres termes ont été inclus dans le modèle d'analyse de certaines variables, comme les effets "date de l'épreuve " et "heure de l'épreuve " pour les températures rectales ou la régression linéaire sur le poids vif de l'animal pour la note de développement musculaire et l'épaisseur de lard dorsal. Afin de préciser la signification statistique de certaines différences, la valeur de combinaisons linéaires appropriées des constantes des moindres carrés a été testée par repport à zéro.

\section{Résultats}

Le tableau 2 donne la proportion de sujets positifs dans les sept échantillons. Les fréquences les plus élevées, de l'ordre de 40 à $50 \mathrm{p}$. Ioo, sont trouvées dans les échantillons $\mathrm{LB}, \mathrm{PB}-\mathrm{F} / 2$ et $\mathrm{PB}-\mathrm{F}_{3}$ qui ne diffèrent pas significativement entre eux de ce point de vue. En ce qui concerne le Piétrain, la fréquence est plus faible dans 1a lignée A (autour de $30 \mathrm{p}$. IOo) que dans la lignée $\mathrm{B}$ (autour de $45 \mathrm{p}$. IOo). La fréquence est de $18 \mathrm{p}$. Ioo chez le Landrace Français et aucun sujet positif n'a été trouvé dans notre échantillon de porcs Large White.

Quant aux cas de mortalité directement consécutifs à l'épreuve d'anesthésie chez les animaux positifs, leur fréquence moyenne dans les populations affectées est de l'ordre de ro p. roo.

Les estimées de la fréquence et de la pénétrance du gène récessif responsable

\section{TABLEAU 2}

Fréquence du syndrome d'hyperthermie maligne $\widehat{(\mathrm{f})}$ et taux de mortalité due à 'épreuve d'anesthésie chez les sujets positits (m) dans les 7 échantillons

Frequency $(\widehat{\mathrm{f}})$ of MHS and mortality rate $(\mathrm{m})$ due to the test among MHS pigs in the 7 samples

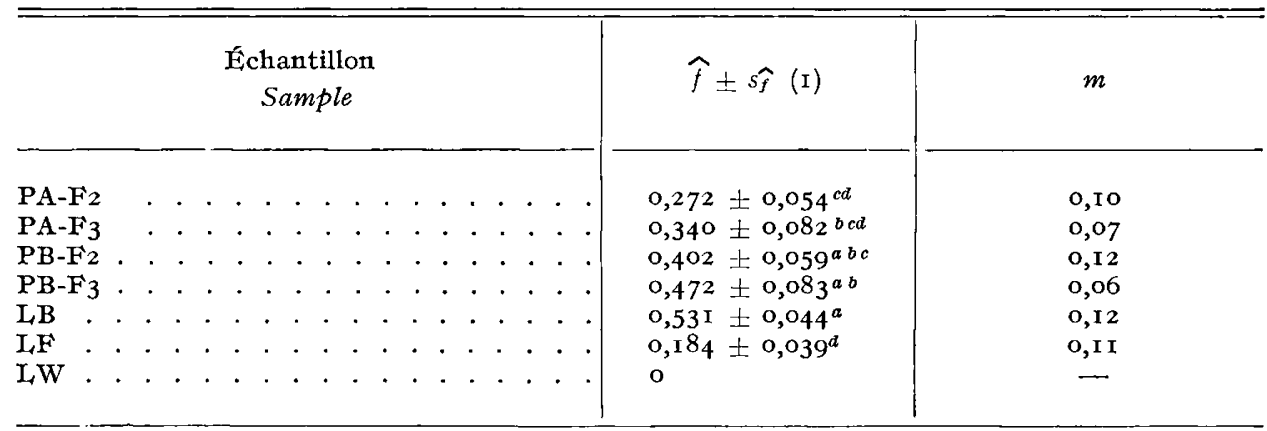

(I) $\widehat{s_{f}}=$ erreur-standard de $\widehat{f}$ (standard error of $\left.\widehat{f}\right)$,

deux valeurs de $\hat{f}$ affectées de la même lettre ne diffèrent pas significativement entre elles, au seuil de probabilité de 5 p. 1oo,

two estimates $\widehat{\mathbf{f}}$ with the same superscript are not significantly different (at the 5 p. roo probability level). 
TABLEAU 3

Fréquence $\widehat{(\mathrm{q})}$ et pénétrance $(\widehat{\mathrm{w}})$ du gène récessif responsable du SHM dans las populations affectées Frequency $\widehat{(\mathrm{q})}$ and penetrance $(\widehat{\mathrm{w}})$ of the postulated recessive "MHS" gene in the affected populations

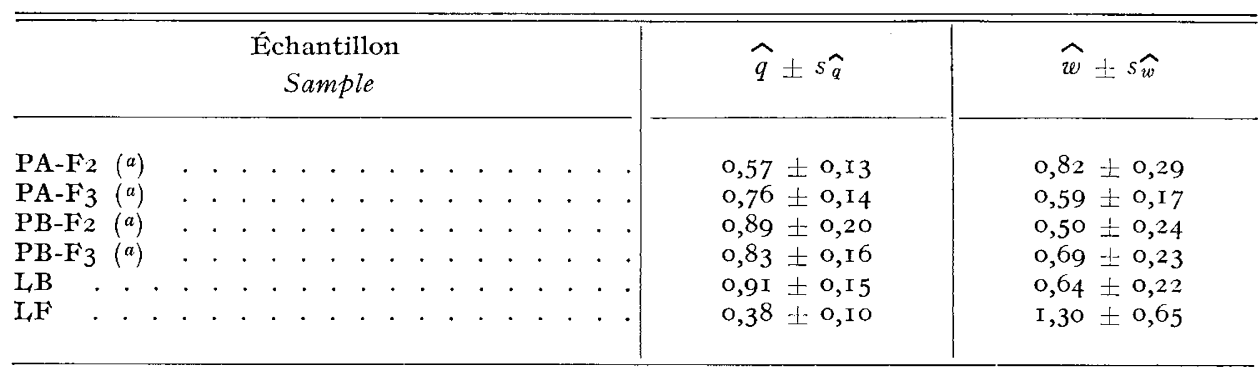

(a) I a variance de dérive est prise en compte dans le calcul de $\widehat{s_{\boldsymbol{q}}}$. Drift variance is included in $\widehat{s_{\boldsymbol{q}}}$.

\section{TABLEAU 4}

Évolution de la température rectale chez les porcs positifs et négatifs et temps moyen de réaction des porcs positifs

Evolution of the rectal temperature in MHS $(+)$ and non-MHS $(-)$ pigs and average reaction time of MHS pigs

\begin{tabular}{|c|c|c|c|c|c|c|}
\hline \multirow{2}{*}{$\begin{array}{l}\text { Variable } \\
\text { Trait }\end{array}$} & \multicolumn{5}{|c|}{$\begin{array}{l}\text { Température rectale }\left({ }^{\circ} \mathrm{C}\right) \\
\text { Rectal temperature }\left({ }^{\circ} \mathrm{C}\right)\end{array}$} & \multirow{2}{*}{$\begin{array}{c}\text { Temps moyen } \\
\text { de réaction } \\
\text { des porcs } \\
\text { positifs (sec.) } \\
\text { Average reaction time } \\
\text { of } M H S \text { pigs (sec.) }\end{array}$} \\
\hline & \multicolumn{2}{|c|}{$\begin{array}{l}\text { Initiale } \\
\text { Initial }\end{array}$} & \multicolumn{3}{|c|}{$\begin{array}{l}\text { Finale } \\
\text { Final }\end{array}$} & \\
\hline $\begin{array}{l}\text { Type de réaction. . . } \\
\text { Type of reaction }\end{array}$ & $(-)$ & $(a)$ & $(-)$ & $(+)$ & (a) & \\
\hline$\widehat{\mathrm{PA}-\mathrm{F} 2}$ et $\mathrm{PB}-\mathrm{F}_{2}\left({ }^{b}\right)$ & $\begin{array}{r}39,89 \\
\pm \quad 0,04 \\
\end{array}$ & $\begin{array}{r}39,93 \mathrm{~ns} \\
\pm \quad 0,05\end{array}$ & $\begin{array}{r}40,01 \\
\text { स. } 0,04\end{array}$ & $\begin{array}{r}40,4 \mathrm{I} \\
\pm \quad 0,05\end{array}$ & & II5 $59(n=73)$ \\
\hline 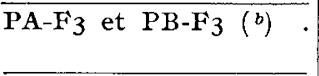 & $\begin{array}{r}39,95 \\
\pm \quad 0,04 \\
\end{array}$ & $\begin{array}{r}40,07 \\
\pm \quad 0,04 \\
\end{array}$ & $\begin{array}{r}39,97 \\
\pm \quad 0,04 \\
\end{array}$ & $\begin{array}{r}40,4 \mathrm{I} \\
\pm \quad 0,04 \\
\end{array}$ & & $85 \pm 7(n=123)$ \\
\hline$\cdot \cdot \cdot \cdot \cdot \cdot \cdot \cdot \cdot \cdot$ & $\begin{array}{r}39,64 \\
\vdots \quad 0,09\end{array}$ & $\begin{array}{r}39,72 \quad \mathrm{~ns} \\
\pm \quad 0,08\end{array}$ & $\begin{array}{r}39,80 \\
+\quad 0,08\end{array}$ & $\begin{array}{r}39,97 \\
\pm \quad 0,08\end{array}$ & - & I36 \pm I I $(n=68)$ \\
\hline $\begin{array}{l}\mathrm{LF} \cdot \cdot \cdot \cdot \cdot \cdot \cdot \cdot \cdot \cdot \cdot \\
\mathrm{LW} \cdot \cdot \cdot \cdot \cdot \cdot \cdot \cdot \cdot \cdot \cdot\end{array}$ & $\begin{array}{r}39,37 \\
\pm \quad 0,07 \\
39,48 \\
\pm \quad 0,07\end{array}$ & $\begin{array}{r}39,27 \\
\pm \quad 0, \mathrm{II} \\
\end{array}$ & $\begin{array}{r}39,43 \\
\pm \quad 0,07 \\
39,53 \\
\pm \quad 0,07\end{array}$ & $\begin{array}{r}39,69 \\
+\quad 0, \text { I I } \\
-\end{array}$ & * & I $59 \pm 25(n=\mathrm{x} 8)$ \\
\hline
\end{tabular}

(a) Signification statistique de la différence entre $(+)$ et $(-) \quad$, ns $\mathrm{P}>$ 0,10 $* \mathrm{P}<0,05$. Statistical significance of the difference between $(+)$ and $(-)\} \S \mathrm{P}<0, \mathrm{ro} * * \mathrm{P}<0, \mathrm{or}$.

(b) Dans ces deux échantillons et pour les deux températures, il n'y a pas de différence significative entre femelles et mâles, ni d'interaction significative type de réaction $\times$ lignée et type de réaction $\times$ sexe; l'effet sexe n'est pas significatif pour le temps moyen de réaction des porcs positifs. In these two samples and for the two temperatures, there are neither significant difference between females and males nor significant interaction between type of reaction and line or sex; there is no sex effect on the average reaction time of $(+)$ pigs. 
Ann. Génét. Sél. anım., I978, 10 (2), I9I-208.

\section{Erratum}

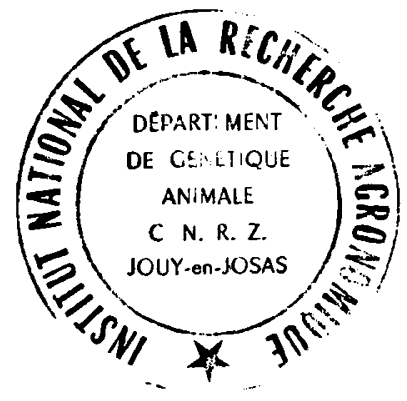

\section{Fréquence du syndrome d'hyperthermie maligne dans des populations porcines françaises; relation avec le développement musculaire}

L. OLLIVIER, P. SELLIER et G. MONIN(*)

avec la collaboration technique de Marie-Reine Langl,ois, P. DaNDo, C. FrLgines, A. Talmant (*), D. TASTU et P. VERNIN (*)

Station de Génétique quantitative et appliquée, Centre national de Recherches zootechniques, I.N.R.A., 78350 Jouy-en-Josas

(*) Station de Recherches sur la viande, Centre de Recherches de Clermont-Ferrand, I.N.R.A., Theix, 63IIo Beaumont

Page r97: $3^{\mathrm{e}}$ ligne et suivantes lire :

...Pour le Large White, la limite supérieure de l'intervalle de confiance de la fréquence génique, correspondant à une probabilité inférieure a 5 p. 100 de ne trouver aucun positif dans l'échantillon étudié, est égale à 0,17 en supposant une pénétrance complète. Les estimations... 

du SHM sont données dans le tableau 3. La fréquence génique est à peu près la même $(0,8-0,9)$ chez le Landrace Belge et dans la lignée B de Piétrain. Elle est un peu plus faible dans la lignée A de Piétrain et nettement plus faible chez le Landrace Français. Pour le Large White, la limite supérieure de l'intervalle de confiance de la fréquence génique, correspondant à une probabilité inférieure à $5 \mathrm{p}$. roo de trouver au moins un positif dans l'échantillon étudié, est égale à 0,022 en supposant une pénétrance complète. Les estimations de la pénétrance du gène sont très peu précises, surtout pour l'échantillon Landrace Français, mais il semble que cette pénétrance est plus forte dans cette dernière race que chez le Piétrain et le Landrace Belge où les valeurs trouvées sont comprises entre 0,5 et 0,8 .

Le tableau 4 rapporte les valeurs moyennes de la température rectale, initiale et finale, chez les deux types de porcs. La différence entre positifs et négatifs n'est pas significative pour la température rectale au début de l'épreuve, sauf dans le cas des animaux Piétrain de la génération $\mathrm{F}_{3}$. Par contre et quelle que soit la population considérée, la température rectale finale est, comme il était prévisible, significativement plus élevée chez les porcs positifs : 1'accroissement moyen de température rectale chez ces derniers varie, selon la population, entre 0,25 et $0,48^{\circ} \mathrm{C}$. Le " temps de réaction " des sujets positifs, mesuré à compter du début de 1'anesthésie effective, est très variable, l'écart-type variant entre 73 et I04 secondes selon la population. En moyenne, ce temps de réaction est plus faible chez le Piétrain que dans les deux populations de Landrace, en particulier le Landrace Français (tab1. 4).

La valeur moyenne de la note de développement musculaire est donnée au tableau 5 pour chaque type de réaction et pour chaque échantillon. Les animaux

TABLEAU 5

Valeurs moyennes, par type de réaction, de la note de développement musculaire dans les sept échantillons

Means (土 s.e.) for score of muscular development according to type of reaction to halothane anesthesia in the seven samples

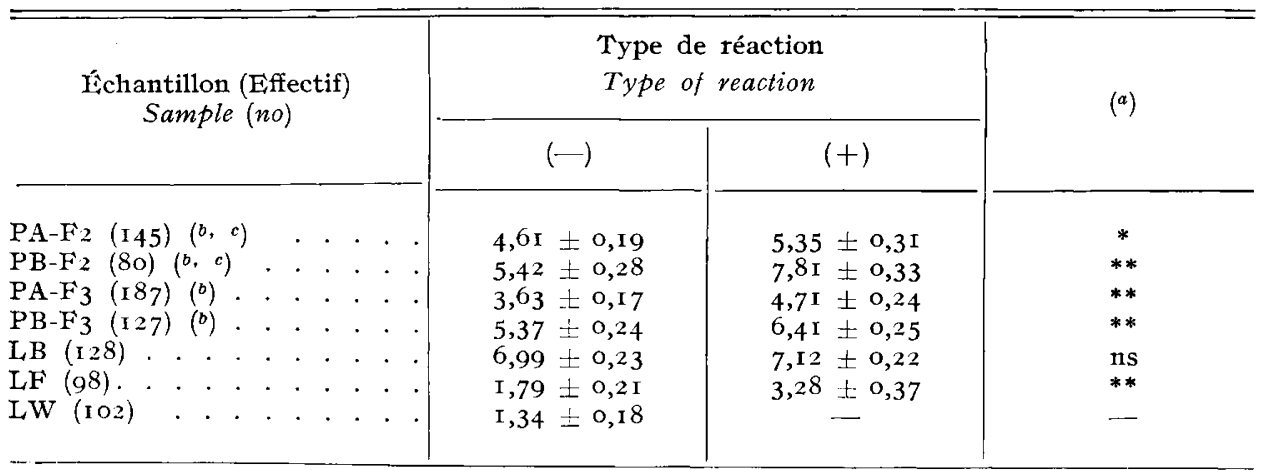

(a) Voir tableau 4 (as in table 4 ).

(b) Dans ces quatre échantillons, il n'y a pas d'effet du sexe de l'animal, ni d'interaction sexe $\times$ type de réaction pour la note de conformation.

In these four samples, there are no effect of sex and no sex $\times$ type of reaction interaction for conformation score.

(c) Dans la génération $\mathrm{F}_{2}$, l'interaction lignée $\times$ type de réaction est hautement significative $(\mathrm{P}<\mathrm{O}, \mathrm{OI})$.

In the $F_{2}$ generation, the line $\times$ type of reaction interaction is significant at the o.or level. 
positifs ont une note significativement plus forte que les animaux négatifs dans les quatre échantillons Piétrain et dans l'échantillon Landrace Français. Par contre, la différence est beaucoup plus faible et non significative dans l'échantillon Landrace Belge.

La relation entre le pourcentage de sujets réagissants dans les 5 populations et la valeur moyenne de la note de développement musculaire est illustrée par la figure I : le classement des populations (LW, LF, PA, PB, LB dans l'ordre croissant) est le même pour ces deux variables.

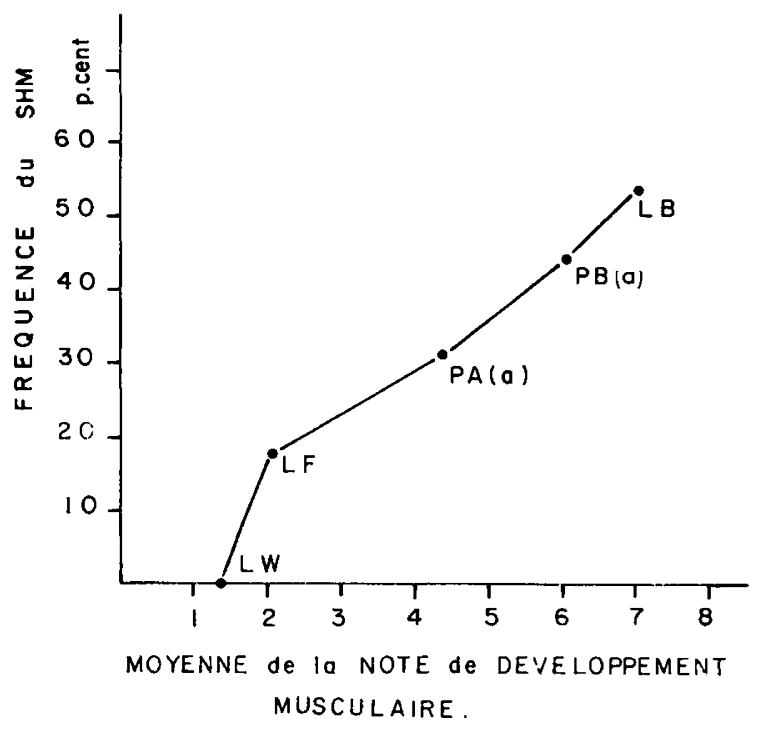

FIG. I. - Relation entre fréquence du syndrome d'hyperthermie maligne et moyenne de la population pour la note de développement musculaire.

Relationship between frequency of MHS and mean score of muscular development.

(a) pour les populations $\mathrm{PA}$ et $\mathrm{PB}$, les données relatives aux générations $\mathrm{F} z$ et $\mathrm{F}_{3}$ sont regroupées.

for $P A$ and $P B$, data on $F_{2}$ and $F_{3}$ generations are pooled.

Les différences entre les deux types de porcs sont données dans le tableau 6 pour les performances d'engraissement et l'épaisseur de lard dorsal.

La différence de gain moyen quotidien est, dans tous les cas, à l'avantage des porcs négatifs et d'importance à peu près égale (entre ro et $20 \mathrm{~g} /$ jour). Elle n'atteint le seuil de signification de $5 \mathrm{p}$. Ioo que dans le cas des porcs Piétrain de la génération $\mathrm{F}_{3}$; encore faut-il préciser que dans ce dernier cas l'interaction lignée $\times$ type de réaction est hautement significative $(\mathrm{P}<0, \mathrm{or})$ et que 1 'avantage des porcs négatifs pour le gain moyen quotidien est observé dans la seule lignée $\mathrm{B}(+45 \mathrm{~g} / \mathrm{jour})$. Cette interaction n'a pas été trouvée à la génération $F 2$. Pour 1'indice de consommation qui a été mesuré seulement chez les animaux LF et $L_{1} B$, aucun effet du type de réaction n'est mis en évidence.

Les sujets positifs présentent une épaisseur moyenne de lard dorsal plus faible (- 0,7 à $-0,8 \mathrm{~mm})$ : cet avantage est statistiquement significatif chez le Landrace Belge $(\mathrm{P}<0,05)$ et chez les porcs Piétrain de la génération $\mathrm{F} 3(\mathrm{P}<0,0 \mathrm{r})$ et il 
est très proche du seuil de signification de 5 p. Ioo chez le Landrace Français. Il apparaît que chez le Piétrain et chez le Landrace Belge, la différence d'épaisseur de lard entre positifs et négatifs est surtout marquée au niveau du " rein ". Dans le cas des porcs Piétrain de la génération $\mathrm{F}_{3}$, une interaction significative entre le sexe et le type de réaction se manifeste pour les trois mesures d'épaisseur de lard et pour leur moyenne : pour cette dernière, la différence entre positifs et négatifs atteint presque $I, 5 \mathrm{~mm}$ chez les femelles mais elle est pratiquement nulle chez les mâles; par contre, l'interaction sexe $\times$ type de réaction est tout à fait mineure pour ces mêmes variables chez les porcs Piétrain de la génération F2.

\section{TABLEAU 6}

Différences entre les deux types de porcs ( $d=$ positifs - négatifs) pour les caractères de croissance et l'épaisseur du lard dorsal

Differences between the two types of pigs ( $d=$ positive - negative) for growth traits and ultrasonic backfat thickness

\begin{tabular}{|c|c|c|c|c|c|}
\hline \multirow{2}{*}{\multicolumn{2}{|c|}{$\begin{array}{l}\text { Caractère } \\
\text { Trait }\end{array}$}} & \multicolumn{4}{|c|}{$\begin{array}{c}\text { Échantillon (Effectif) } \\
\text { Sample (no) }\end{array}$} \\
\hline & & $\begin{array}{c}\mathrm{PA}^{-\mathrm{F}_{2}} \text { et } \\
\mathrm{PB}-\mathrm{F}_{2} \\
(\text { I } 79)\end{array}$ & $\begin{array}{c}\mathrm{PA}-\mathrm{F}_{3} \text { et } \\
\mathrm{PB}-\mathrm{F}_{3} \\
(27 \mathrm{I})\end{array}$ & $\begin{array}{l}\mathrm{LB} \\
(\mathrm{I} I \mathrm{I})\end{array}$ & $\begin{array}{l}\text { LF } \\
(94)\end{array}$ \\
\hline \multicolumn{2}{|c|}{$\begin{array}{l}\text { Gain moyen quotidien (g) } \\
\text { Average daily gain }(g) \\
\text { Indice de consommation }(\mathrm{kg} \\
\quad \text { aliment } / \mathrm{kg} \text { gain) } \cdot \cdot^{\circ} \cdot \cdot \cdot \cdot \\
\text { Food conversion (kg feed } / \mathrm{kg} \\
\quad \text { gain) }\end{array}$} & 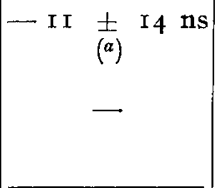 & $\begin{array}{c}- \text { I9 } \underset{(b)}{\frac{ \pm}{(b)}} 8 * \\
-\end{array}$ & 一 9 土 $\mathrm{I} 4 \mathrm{~ns}$ & $\begin{array}{l}-\mathrm{I} 5 \pm 2 \mathrm{Ins} \\
-0,04 \pm 0,05 \mathrm{~ns}\end{array}$ \\
\hline $\begin{array}{l}\text { Épaisseur } \\
\text { de lard } \\
\text { Backfat } \\
\text { thickness } \\
\text { (mm) }\end{array}$ & $\begin{array}{l}\text { Rein } \\
\text { Rump } \\
\text { Dos } \\
\text { Last rib } \\
\text { Cou } \\
\text { Shoulder } \\
\text { Moyenne } \\
\text { Average }\end{array}$ & $\begin{array}{l}-\mathrm{I}, 0 \pm 0,5^{*} \\
-0,3 \pm 0,3 \mathrm{~ns} \\
-0,7 \pm 0,6 \mathrm{~ns} \\
-0,7 \pm 0,4 \mathrm{~ns}\end{array}$ & $\begin{array}{l}-\mathrm{I}, 0 \underset{(c)}{ \pm} 0,2^{* *} \\
-0,5 \underset{(c)}{ \pm} 0,2^{*} \\
-0,6 \underset{(c)}{ \pm} 0,4 \mathrm{~ns} \\
-0,7 \underset{\frac{(c)}{(c)}}{ \pm} 0,2\end{array}$ & $\begin{array}{l}-\mathrm{r}, 0 \pm 0,3^{* *} \\
-0,6 \pm 0,3^{*} \\
-0,5 \pm 0,3 \mathrm{~ns} \\
-0,7 \pm 0,3^{*}\end{array}$ & $\begin{array}{l}-0,4 \pm 0,5 \mathrm{~ns} \\
-0,9 \pm 0,4^{*} \\
-\mathrm{I}, 0 \pm 0,5^{*} \\
-0,8 \pm 0,4 \S\end{array}$ \\
\hline
\end{tabular}

(a) $d \pm s_{d}$.

(b) Interaction lignée $\times$ type de réaction significative $(\mathrm{P}<0$,or $)$. Line $\times$ type of reaction interaction significant at the $0.0 \mathrm{I}$ level.

(c) Interaction sexe $x$ type de réaction significative $(\mathrm{P}<0,05)$. Sex $\times$ type of reaction interaction significant at the 0.05 level.

\section{Discussion}

Les résultats qui viennent d'être présentés appellent un certain nombre de commentaires, tant du point de vue de la variation entre populations de la fréquence des sujets manifestant le syndrome d'hyperthermie maligne lors de l'épreuve d'anesthésie à l'halothane que du point de vue des liaisons entre la sensibilité à 
J'halothane et les caractères de production, notamment le développement musculaire de l'animal.

La température rectale notée en fin d'épreuve est significativement plus élevée chez les sujets positifs que chez les sujets négatifs, la différence variant entre $0, \mathbf{I} 7$ et $0,44^{\circ} \mathrm{C}$ selon 1'échantillon considéré. Ces chiffres sont du même ordre de grandeur que la valeur de $0,3^{\circ} \mathrm{C}$ trouvée par $\mathrm{WEBB}$ et JoRdAN (I977) chez les porcs d'une lignée synthétique à base de Piétrain et de Hampshire. Les données relatives au Piétrain montrent que, dans le cas d'une réaction positive, l'accroissement de la température rectale en cours d'épreuve (de l'ordre de $0,4^{\circ} \mathrm{C}$ ) et la durée de l'épreuve ne diffèrent pas de façon significative entre mâles et femelles; dans notre première étude concernant des femelles et des mâles castrés de cette même race (MonIN et al., I976), l'accroissement de la température rectale avait été nettement moins marqué chez les mâles castrés positifs que chez les femelles positives et la détection de la réaction positive avait été plus précoce chez ces dernières. Dans l'étude de WEBB et JORDAN (I978), le temps moyen de réaction des sujets positifs est égal à 9r secondes et donc sensiblement inférieur aux temps observés dans la présente étude et dans celle de Monin et al. (I976), si l'on tient compte du fait que le temps est compté à partir du début de l'administration d'halothane et non pas, comme nous le faisons, à partir du début de l'anesthésie effective; cette différence peut s'expliquer par la plus forte concentration initiale en halothane du mélange gazeux (jusqu'à 8 p. Ioo) dans la technique d'anesthésie utilisée par WEBB et JORDAN (I978).

Ces mêmes auteurs ont, par ailleurs, enregistré un taux de mortalité due au test à 1'halothane chez les sujets réagissants égal à 8,6 p. Ioo et donc tout à fait comparable au taux moyen enregistré dans notre étude. Par contre ce taux est beaucoup plus faible ( $\mathrm{I}$ p. Ioo environ) dans les expériences réalisées sur le Landrace Néerlandais (EIKELENBOom et al., I977) et sur les Landrace et Yorkshire Suisses (SCHWÖRER et BLUM, I977).

L'un des enseignements majeurs de notre étude est l'existence de grandes différences entre les races couramment exploitées en France quant à la proportion d'animaux sensibles à l'halothane.

L'absence de sujets réagissants dans notre échantillon de la race Large White est en accord avec les résultats trouvés dans une lignée de Yorkshire Néerlandais par Cöp et Buitring (I976) et chez le Large White Irlandais par Mc GLoughirn et al. (I977). Notons toutefois que dans une étude à grande échelle de la population Yorkshire Néerlandais, 3 p. Ioo des I304 animaux soumis au test à l'halothane ont été reconnus positifs (EIKELENBOOM et al., I978) et que la proportion de réagissants atteint même r3,4 p. Ioo chez le Yorkshire Suisse (ScHwörER et BLUM, I977) : cette dernière valeur, un peu surprenante, a été obtenue sur un échantillon d'une taille appréciable puisqu'il comprend 209 porcs issus de 48 portées. D'une façon générale, du point de vue de la fréquence des animaux sensibles à l'halothane, la race Large White (ou Yorkshire) est à rapprocher des races Duroc et Hampshire où cette fréquence est nulle ou très faible (moins de $2 \mathrm{p}$. IOO), d'après les observations de Riggs et al. (I974), Cöp et Buiting (I976) et WEBB et JoRdan (I978); un pourcentage plus élevé de positifs (7 p. IOo) a cependant été trouvé par WATANABE (I977, communication personnelle) dans un échantillon de ro8 porcs Duroc. Indiquons également que le gène responsable du SHM est présent chez le Yorkshire Américain à une fréquence, semble-t-il, non négligeable (CAMPION et al., I975; RASMUSEn et Christian, I976).

La fréquence de réagissants trouvée dans notre échantillon de Landrace Français (I 8,4 p. IOo) est sensiblement supérieure aux fréquences trouvées dans plusieurs autres populations de Landrace de type "traditionnel »:4,5 p. Ioo pour 
le Landrace Norvégien (WEBB et JoRDAN, I978), 4,8 p. Ioo pour le Landrace Irlandais (Mc Gloughlin et al., I977), 9,2 p. Ioo pour le Landrace Danois (JørGensen, I977). Par contre, elle est comparable à la fréquence de 22,2 p. Ioo trouvée par EIKELENBOOM et al. (I978) sur un effectif important ( $n=\mathrm{I} 640)$ de porcs Landrace Néerlandais envoyés dans les stations de sélection, c'est-à-dire dans des conditions d'échantillonnage proches des nôtres. Chez le Landrace Suisse, la proportion de réagissants est égale à 24,3 p. IOO, d'après les résultats de SchwöRER et BLUM (I977). La variabilité des estimées rapportées ci-dessus s'explique bien sûr en partie par la relative imprécision de certaines d'entre elles, liée à la fois à la taille réduite et à la plus ou moins bonne représentativité des échantillons étudiés. Il semble toutefois que même en mettant à part le Landrace Belge, il existe une réelle variation de la fréquence des animaux sensibles à l'halothane entre les diverses "variétés nationales" de Landrace en Europe.

La fréquence des sujets positifs dans notre échantillon d'animaux Landrace Belge est très supérieure à celle du Landrace Français. La fréquence observée ici (53,I p. I0o) reste toutefois beaucoup plus faible que la fréquence de 84,8 p. Ioo trouvée par Cöp et Buiting (I976) dans un autre échantillon de Landrace Belge. Remarquons que cette dernière valeur a été obtenue, sur un nombre relativement réduit d'animaux $(n=50)$ dans une lignée expérimentale et non pas, comme ici, sur un échantillon d'animaux provenant de I6 élevages de sélection de la race et représentant 80 portées issues de 49 pères différents. En dépit de la représentativité satisfaisante de l'échantillon, nous sommes même portés à croire, pour la raison développée plus loin, que le chiffre de $53, \mathrm{I}$ p. Ioo est une légère surestimation de la fréquence vraie des réagissants dans la population Landrace Belge exploitée en France.

La fréquence trouvée dans notre lignée A de Piétrain, de l'ordre de 30 p. Ioo, est comparable à la valeur observée ( $28 \mathrm{p}$. roo) dans un premier échantillon de cette race (Olifivier et al., I975). Dans la lignée B de Piétrain sélectionnée pour un fort développement musculaire, la proportion de réagissants est notablement plus élevée puisqu'elle avoisine 40-45 p. roo dans les deux générations considérées ici $\left(\mathrm{F}_{2}\right.$ et $\left.\mathrm{F}_{3}\right)$ et qu'elle atteint $73 \mathrm{p}$. Ioo à la génération de sélection suivante $\left(\mathrm{F}_{4}\right)$. Dans une lignée Piétrain entretenue aux Pays-Bas, Cöp et Buiting (I976) ont obtenu roo p. roo de réactions positives alors que dans le troupeau Piétrain de 1'Université du Minnesota, la fréquence des sujets sensibles à l'halothane est supérieure à 80 p. Ioo (Eilizondo et al., I976).

A propos de nos estimations de fréquence génique et de pénétrance, un premier point à discuter concerne la validité de l'application de la méthode de LEFORT et al. (I975) à l'ensemble des données que nous avons recueillies. Le principe de cette méthode est de combiner, pour l'estimation de la fréquence génique, l'information relative aux animaux testés pour l'anomalie étudiée et celle relative à leurs parents (transmetteurs ou pas) : l'hypothèse est donc faite que ces parents n'ont été soumis, entre la naissance et la mise à la reproduction, à aucune sélection directe ou indirecte vis-à-vis de l'anomalie. La sélection naturelle contre l'anomalie (mortalité due au syndrome de stress) est, semble-t-il, de faible intensité dans les conditions normales d'élevage des futurs reproducteurs. Par contre, et a priori ceci vaut en particulier pour la lignée B de Piétrain et pour le Landrace Belge, la possibilité d'une sélection artificielle en faveur du gène responsable du SHM dans la génération des parents ne peut être exclue, avec comme conséquence une surestimation de la fréquence génique dans ces cas-là.

En ce qui concerne la pénétrance du gène chez les homozygotes, les estimées obtenues dans les quatre échantillons Piétrain sont en accord avec la valeur de 
$0,69 \pm 0,25$ obtenue dans notre précédent échantillon de porcs de la même race (Olifivier et al., I975). Deux des estimées de la présente étude (PB-F2 et PA-F3) diffèrent significativement de $\mathrm{I}$ et on peut conclure à une pénétrance incomplète, de l'ordre de 0,6-0,7, chez le Piétrain et aussi, semble-t-il, chez le Landrace Belge. Il apparaît d'autre part que la pénétrance est plus faible dans ces deux races que chez le Landrace Français. La très grande imprécision de l'estimée relative à cette dernière population ne permet pas de voir là plus qu'une présomption de l'existence de différences réelles entre races de ce point de vue. Cette situation pourrait être la conséquence d'une sélection en faveur d'une faible pénétrance du gène responsable du SHM dans les deux races à fort développement musculaire, une telle sélection n'ayant pas été aussi efficace chez le Landrace Français où le gène a été introduit plus récemment. Les autres données de la littérature s'accordent pour montrer que la pénétrance du gène chez les homozygotes est très proche de ou égale à I. Dans une lignée synthétique à base de Piétrain et de Hampshire, Smiтн et BAMPTON (I977) estiment cette pénétrance à 0,95. Chez le Landrace Néerlandais, MrNKEMA et al. (I977) concluent que leurs résultats sont compatibles avec 1'hypothèse d'une pénétrance complète; ANDRESEN et JENSEN (I977) concluent également à une pénétrance élevée dans le cas d'accouplements entre hétérozygotes chez le Landrace Danois. Remarquons en fin que notre hypothèse de pénétrance incomplète chez le Piétrain est évidemment peu compatible avec le fait que Cöp et BuITING (I976) n'aient trouvé que des animaux positifs $(n=53)$ dans une lignée de cette race. Dans la méthode d'estimation utilisée ici, 1'hypothèse est faite que la pénétrance du gène récessif responsable de l'anomalie est d'origine non génétique. Sous cette hypothèse, la pénétrance incomplète pourrait s'expliquer, dans le cas présent, par la durée relativement limitée de l'épreuve d'anesthésie, d'où la nondétection de certains réagissants " potentiels " chez lesquels les premiers symptômes caractéristiques du SHM seraient apparus au-delà des 5 minutes retenues dans cette expérience. Il est indéniable que cette explication vaut au moins pour quelques cas : signalons à titre d'exemple que, la durée de l'épreuve ayant été portée à 20 minutes chez 33 porcs Piétrain reconnus négatifs au bout de 5 minutes, nous avons obtenu une réaction positive, après une dizaine de minutes, chez deux d'entre eux. I1 n'empêche que nous avons des raisons de penser que la pénétrance, telle que nous l'apprécions avec l'épreuve de 5 minutes, a une part génétique; la méthode appliquée ici conduit, nous l'avons vu, à des estimations de pénétrance avoisinant 0,7 pour le Piétrain alors que dans I 7 portées issues de parents positifs de cette race, I02 des I09 porcs testés ont été reconnus positifs, ce qui correspond à une pénétrance de 93.5 p. Ioo pour ce type d'accouplement. On peut imaginer que les individus manifestant le syndrome avec l'épreuve utilisée sont porteurs de gènes modificateurs favorisant l'expression du syndrome et transmis à leurs descendants et par conséquent que la pénétrance est plus forte chez les homozygotes provenant d'accouplements entre positifs que chez les homozygotes provenant d'autres types d'accouplement.

En ce qui concerne la relation entre le type de réaction à l'halothane et le gain moyen quotidien, les résultats de notre étude concordent pour les différentes populations et vont dans le sens d'un léger désavantage, le plus souvent non significatif, des sujets positifs. Dans notre précédente étude sur le Piétrain (Monin et al., I976) et dans 1'étude de WEBB et JoRDAN (I978), la différence entre les deux types de porcs n'est pas significative mais, contrairement à ce qui est trouvé ici, la tendance est en faveur des sujets positifs. Dans les diverses comparaisons rapportées par EIKELENBOOM et al. (I977, I978) et concernant les Landrace et Yorkshire Néerlandais, la plupart des différences ne sont pas significatives mais certaines 
différences en faveur des sujets négatifs atteignent le seuil de signification statistique : c'est en particulier le cas en alimentation à volonté où la différence de gain moyen quotidien approche $50 \mathrm{~g} /$ jour et est à relier à une différence significative de consommation moyenne journalière. Toutefois, chez le Landrace Suisse et également en alimentation à volonté, GERwiG et al. (I977) ne trouvent pas de différence significative entre les deux types de porcs pour la vitesse de croissance. Par ailleurs, comme nous l'avons observé ici pour le Landrace Français et pour 1e Landrace Belge, l'effet du type de réaction à l'halothane sur l'indice de consommation n'est pas significatif dans les études de WEBB et JORDAN (I978) et EI KELEN-. воом et al. (I978). Il ressort de l'ensemble de ces résultats que les performances de croissance et d'efficacité alimentaire ne diffèrent pas notablement entre les deux types de porcs, bien que, dans certaines conditions, les sujets positifs aient une vitesse de croissance plus faible.

Sur le plan de la composition corporelle, nous disposons ici de deux types d'information sur 1'animal vivant : la note de développement musculaire donnée au poids vif de $25-30 \mathrm{~kg}$ et 1'épaisseur de lard dorsal mesurée avec un appareil à ultra-sons au poids vif de $80-85 \mathrm{~kg}$.

La réduction de l'épaisseur de lard dorsal chez les sujets positifs est d'égale importance $(0,7-0,8 \mathrm{~mm})$ dans les différentes races étudiées ici. Un avantage des positifs pour l'épaisseur de lard dorsal mesurée in vivo a été également mis en évidence par GERWIG et al. (I977) et par EIKELENBOom et al. (I977) chez des porcs nourris à volonté. Dans le cas de verrats alimentés semi ad libitum, la réduction de cette épaisseur de lard est de $0,7 \mathrm{~mm}(\mathrm{P}<\mathrm{o}, \mathrm{or})$ chez le Landrace Néerlandais mais chez le Yorkshire Néerlandais, il n'y a pas de différence significative entre positifs et négatifs de ce point de vue (EIKELENBoom et al., I978). En ce qui concerne l'épaisseur de lard dorsal mesurée sur la carcasse, Monin et al. (1976) et WEBB et JORDAN (I978) n'ont pas trouvé d'influence significative du type de réaction à 1'halothane alors qu'un avantage significatif des positifs a été mis en évidence chez les mâles castrés, mais non chez les femelles, dans l'étude d'Eir KELENBOOM et Minkema (I974) surle Landrace Néerlandais. D'après des données recueillies ultérieurement sur un effectif beaucoup plus important de femelles de cette même race (EIKELENBOOM et al., r978), l'effet du type de réaction à l'halothane est hautement significatif, avec un avantage des femelles positives égal à $0,8 \mathrm{~mm}$ alors que cet avantage est plus faible $(0,2 \mathrm{~mm})$ et non significatif chez des femelles Yorkshire Néerlandais. Au total et en dépit de la variabilité des résultats, sans doute liée pour une bonne part au petit nombre de sujets positifs dans certaines comparaisons, on peut conclure à une réduction de 1'épaisseur de lard dorsal chez ces derniers et la situer aux alentours de $0,8 \mathrm{~mm}$. Il semblerait aussi que 1'importance de cette réduction varie selon le site de mesure et que le "profil longitudinal" de la couche de lard dorsal diffère chez les deux types de porcs : les résultats de la présente étude sur le Piétrain et le Landrace Belge, ainsi que ceux de Monin et al. (I976) et d'EIKELENBOOM et MINkEMA (I974), suggèrent que la diminution de l'épaisseur de lard chez les sujets positifs est plus nette au niveau de la région lombaire qu'au niveau de la région dorsale.

Les résultats concernant la note visuelle de développement musculaire sont plus éloquents quant à l'avantage de composition corporelle des porcs positifs. Rappelons d'abord que la méthode de notation utilisée a une signification bien spécifique, en ce sens qu'elle vise à apprécier in vivo sur le jeune animal le degré de manifestation du phénomène d'hypertrophie musculaire caractéristique du Piétrain ou tout au moins des animaux " extrêmes" de cette race. Indiquons aussi, pour situer la signification de cette note en termes de composition de la carcasse, que 
la régression du pourcentage de morceaux nobles (jambon + longe) de l'animal abattu à $90 \mathrm{~kg}$ sur la note de conformation donnée à $20-25 \mathrm{~kg}$ est voisine de $0,5 \mathrm{p}$. Ioo par point chez le Piétrain (données préliminaires non publiées).

Entre populations, on observe une relation étroite entre fréquence des sujets sensibles à l'halothane et note moyenne de développement musculaire. Ceci apparaît clairement quand on compare le Large White, le Landrace Français et le Landrace Belge et quand on compare les deux lignées Piétrain sélectionnées pour des objectifs différents. La lignée $B$ sélectionnée en faveur de l'hypertrophie musculaire présente, vis-à-vis de la lignée $A$, à la fois une plus forte fréquence de sujets positifs et une moyenne plus élevée de la note de conformation, tant à la F2 qu'à la F3. Cette double divergence entre lignées est encore plus nette si l'on prend en compte la génération $\mathrm{F}_{4}$ où l'avantage de la lignée $\mathrm{B}$ pour la note de conformation atteint 2,4 points (contre $\mathrm{I}, 5$ point à la $\mathrm{F}_{2}$ et $\mathrm{I}, 9$ point à la $\mathrm{F}_{3}$ ) et où la fréquence des sujets positifs est I,7 fois plus élevée dans la lignée B (73 p. Ioo) que dans la lignée A (43 p. I00).

La relation entre sensibilité à 1'halothane et développement musculaire se retrouve également à l'intérieur des diverses populations étudiées, à l'exception du Landrace Belge où la différence entre positifs et négatifs n'est pas significative. Cette exception peut s'expliquer par la sélection sur la conformation faite par l'éleveur quand il choisit les jeunes verrats destinés aux stations de contrôle individuel : l'échantillon étudié ne serait donc pas un échantillon aléatoire de la race vis à-vis du développement musculaire, ce que suggère 1'examen de la distribution de fréquence des notes de conformation avec une sous-représentation des notes les plus faibles correspondant pour la plus grande part à des animaux négatifs. L'égal développement musculaire des deux types de porcs dans notre échantillon Landrace Belge montre aussi que des sujets négatifs peuvent présenter un très bon développement musculaire, de tels cas étant également rencontrés dans les échantillons Piétrain. La grande majorité de ces animaux négatifs sont des hétérozygotes au locus de la sensibilité à l'halothane et ceci suggère que le gène responsable de cette sensibilité est, au moins partiellement, dominant sur son allèle " normal " quant à son effet sur le développement musculaire. Cette hypothèse a été précédemment proposée pour expliquer le maintien à des fréquences élevées du gène de sensibilité à l'halothane dans les races à fort développement musculaire (Ol_IVIER et al., I975). L'autre conséquence, déjà évoquée, de ce biais d'échantillonnage dans la population Landrace Belge est une surestimation de la fréquence des réagissants et du gène responsable du SHM dans cette race. Le choix sur conformation par l'éleveur a très probablement une incidence négligeable chez le Landrace Français et chez le Large White, races dans lesquelles un fort développement musculaire n'est pas recherché comme chez le Landrace Belge ou le Piétrain.

L'avantage des animaux positifs pour la note de développement musculaire donnée au poids vif de $25-30 \mathrm{~kg}$ est à rapprocher des résultats obtenus sur la composition corporelle appréciée à l'abattage vers $85-90 \mathrm{~kg}$. Les données recueillies sur ce point dans différentes populations (EIKELENBOom et MinkEMA, I974; Monin et al., I976; EIKELENBOOM et al., I978; WEBB et JORDAN, I978) concordent pour montrer qu'à un poids d'abattage donné, la carcasse des sujets positifs est à la fois plus lourde et plus courte, donc plus "compacte ": les différences de rendement en carcasse et de longueur varient respectivement de 0,5 à $\mathrm{I}, 4$ point et de 0,6 à $2 \mathrm{~cm}$ selon les études. D'autre part la teneur en viande de la carcasse est sensiblement plus élevée chez les sujets positifs que chez. les sujets négatifs. A partir des résultats obtenus chez le Piétrain par Monjn et al. (I976), l'avantage des positifs pour le pourcentage de muscle dans la carcasse peut être évalué à environ 2,5 points au 
poids vif d'abattage de $88 \mathrm{~kg}$; un avantage du même ordre a été trouvé chez le Landrace Suisse (GERWIG et al., I977). Dans une lignée synthétique PiétrainHampshire, WEBB et JORDAN (I978) ont trouvé une supériorité encore plus nette (4 à 5 points) des animaux positifs de ce point de vue; par contre, les différences observées entre positifs et négatifs chez le Landrace Néerlandais et chez le Yorkshire Néerlandais (EIKELENBOom et al., I977, I978) sont, semble-t-il, beaucoup plus faibles. Un dernier point à signaler concerne l'existence éventuelle d'une interaction sexe $\times$ type de réaction pour la composition corporelle : les résultats précédemment obtenus par Monin et al. (I976) et ceux trouvés ici pour l'épaisseur de lard dorsal dans les échantillons $\mathrm{PA}-\mathrm{F}_{3}$ et $\mathrm{PB}-\mathrm{F}_{3}$ tendent à montrer que chez le Piétrain, l'influence du type de réaction à l'halothane sur la composition de la carcasse est plus forte chez les femelles que chez les mâles entiers ou castrés. I1 ne semble pas, au vu des résultats d'EIKELENBoom et al. (I977), que ceci soit le cas chez le Landrace Néerlandais; les premiers résultats concernant cette race (EIKELENBOOM et MINKEMA, I974) indiquent même que la réduction de l'épaisseur de lard dorsal chez les positifs est plus forte pour les mâles castrés que pour les femelles.

\section{Gonclusion}

Cette étude met en évidence des différences très importantes entre les races porcines exploitées en France quant au pourcentage d'animaux sensibles à 1'halothane. Ces différences correspondent à des fréquences variables du gène récessif responsable de cette sensibilité et peut-être aussi à une plus ou moins forte pénétrance du gène chez les homozygotes, ce dernier point n'étant pas encore complètement élucidé. Nos résultats démontrent d'autre part, à la fois entre populations et intra-population, la liaison entre la sensibilité à l'halothane et la composition corporelle, en particulier le degré d'hypertrophie musculaire apprécié visuellement chez le jeune animal. Cette liaison est notamment illustrée, intra-race, par la divergence significative obtenue entre les deux lignées Piétrain pour la fréquence des sujets sensibles à l'halothane, comme conséquence de la sélection de l'une d'elles en faveur de 1'hypertrophie musculaire. Le plus fort développement musculaire des porcs positifs (homozygotes récessifs) étant maintenant établi, il reste à préciser où se situe de ce point de vue l'hétérozygote par rapport aux deux homozygotes. Enfin il importe de noter qu'il subsiste des différences importantes de développement musculaire entre les porcs positifs des races étudiées et donc que la variabilité entre races de ce caractère n'est attribuable qu'en partie au locus du syndrome d'hyperthermie maligne.

Reçu pour publication en juillet 1978 .

\section{Remerciements}

Nous tenons à remercier les éleveurs de l'U.P.R.A. et les Directeurs des stations de sélection de Gannat et du Transloy pour le concours qu'ils ont prêté à cette expérience, ainsi que M. $Z_{F}$ FT, Directeur de l'Institut technique du Porc, pour l'aide matérielle qu'il nous a apportée. 


\title{
Summary
}

\author{
Frequency of the malignant hyperthermia syndrome (MHS) in some French pig \\ populations; relationship with muscular development
}

In 1975 and 1976,872 pigs from five French populations have been tested for MHS susceptibility by means of a 5 -minute halothane anaesthesia, applied over a live weight range of 20 to $35 \mathrm{~kg}$. These populations are : (I) a Piétrain line (PA) selected for high growth rate and low backfat thickness, (2) a Piétrain line (PB) selected for increased muscular hypertrophy, (3) the Belgian Landrace (LB) breed; (4), the French Landrace $(\mathrm{L} F)$ breed and (5) the Large White $(\mathrm{LW})$ breed. In the two experimental Piétrain lines, all male and female piglets weaned have been halothanetested in two successive generations $\left(\mathrm{F}_{2}\right.$ and $\left.\mathrm{F}_{3}\right)$. In the three other breeds ( $\mathrm{L}_{1} \mathrm{~B}, \mathrm{LF}_{1}, \mathrm{LW}_{\mathrm{W}}$ ), the test has been applied to young boars entering performance-testing stations and born in several breeding herds. The numbers of pigs tested are $\mathrm{I}_{47}\left(\mathrm{~F}_{2}\right)$ and I $88\left(\mathrm{~F}_{3}\right)$ for PA, 82 and I 27 for PB, I 28 for $L B, 98$ for $L F$ and $I_{02}$ for $L W$. Muscular development has been assessed visually at the time of the test. Average daily gain and ultrasonic backfat thickness (at $80-85 \mathrm{~kg}$ liveweight) of all pigs and food conversion of individually fed LB, LF and LW pigs have also been recorded.

The frequency ( 1 s.e.) of positive reaction to halothane is $27.2 \pm 5.4,34.0 \pm 8.2,40.2 \pm$ $5.9,47.2 \pm 8.3,53 . \mathrm{I} \pm 4.4, \mathrm{I} 8.4 \pm 3.9$ and $\mathrm{o}$ p. Ioo respectively in $\mathrm{PA}-\mathrm{F}_{2}, \mathrm{PA}-\mathrm{F}_{3}, \mathrm{~PB}_{-} \mathrm{F}_{2}$ $\mathrm{PB}_{3}, \mathrm{LB}, \mathrm{LF}$ and $\mathrm{LW}$ samples. About Io p. Ioo of those reacting to halothane died as a direct result of the test. Assuming as it is now established that MHS is due to a single autosomal recessive gene, the frequency of this gene and its penetrance in homozygotes can be estimated in a population from the distribution of affected animals over the litters tested. The estimates in the PA-F2, PA-F $3, \mathrm{~PB}-\mathrm{F}_{2}, \mathrm{~PB}-\mathrm{F}_{3}, \mathrm{LB}$ and $\mathrm{LF}$ samples are respectively $0.57 \pm 0 . \mathrm{I}_{3}, 0.76 \pm 0.14$, $0.89 \pm 0.20,0.83 \pm 0.16,0.9 \mathrm{I} \pm 0.15$ and $0.38 \pm 0.10$ for gene frequency, and $0.82 \pm 0.29$, $0.59 \pm 0.17,0.50 \pm 0.24,0.69 \pm 0.23,0.64 \pm 0.22$ and $\mathrm{I} .30 \pm 0.65$ for penetrance. Therefore the population differences in incidence of MHS correspond to different frequencies and also possibly to a variable penetrance of the recessive gene responsible for the abnormality. A slight upward bias is likely in the LB gene frequency estimation, as a result of a possible within-litter selection on conformation by the breeder. There is no significant effect of type of reaction to halothane on growth traits though average daily gain of MHS pigs tends to be lower (by around I $5 \mathrm{~g} / \mathrm{day})$ in all samples. The lower average backfat thickness of MHS pigs (0.7-0.8 $\mathrm{mm})$ is generally significant. Considering population means, the incidence of MHS is closely correlated with the score for apparent muscular development. The advantage of MHS pigs for this score is significant in $\mathrm{PA}, \mathrm{PB}$ and $\mathrm{LF}$ populations but not in the $\mathrm{LB}$, probably because of the non-randomness of this sample as explained above. Altogether these results show the strong genetic association between MHS and muscular development; within breed, this association is particularly well examplified by the divergence obtained between the two Piétrain lines for MHS frequency as a consequence of selection for muscular hypertrophy in one of these lines. However large differences which cannot be attributed to the MHS locus exist between breeds with respect to muscular development.

\section{Références bibliographiques}

ANDRESEN E., JENSEN P., I977. Close linkage established between the HAL locus for halothane sensitivity and the PHI (phosphohexose isomerase) locus in pigs of the Danish Landrace breed. Nord. Vet.-Med., 29, 502-504.

ANSAY M., OLLIVIER L., I978. Créatinine plasmatique et sensibilité du porc au syndrome d'hyperthermie maligne. Relations avec deux enzymes du globule rouge (PHI et 6-PGD). Ann. Génét. Sél. anim., 10, 9-16.

Campion D. R., Olson J. C., Topel D. G., Christrian L. L., Kuhlers D. L., i975. Mitochondrial traits of muscle from stress-susceptible pigs. J.Anim. Sci., 41, I3I4-I3I7.

Christran L. L., 1972. A review of the role of genetics in animal stress susceptibility and meat quality. In Cassens R., Giesler F., Kor,B Q., Proceedings of the Pork Quality Symposium, 9I-I I5, University of Wisconsin, Madison, USA. 
Cöp W. A. G., Buitrng G. A. J., 1976. Contribution possible des races étrangères à l'amélioration génétique du Porc aux Pays-Bas (en néerlandais). Rapport C-30I, I6 p., Institut voor Veeteeltkundig onderzoek "Schoonoord ", Zeist, Pays-Bas (Anim. Breed. Abstr., 45, 50r).

Crow J. F., KImUra M., I97o. An Introduction to Population Genetics Theory. Harper and Row, New-York.

EIKELENBOOM G., Minkema D., I974. Prediction of pale, soft, exudative muscle with a nonlethal test for the halothane-induced porcine malignant hyperthermia syndrome. Tijdschr. Diergeneesk., 99, 42 I-426.

Eikelenboom G., Minkema D., van Eldik P., r977. The application of the halothane test. Differences in production characteristics between pigs qualified as reactors (MHS-susceptible) and non-reactors. In Proc. Third Int. Conf. on Production Disease in Farm animals, Sept. I3-I6, I976, I83-I87, Pudoc, Wageningen, Pays-Bas.

Eikelenboom G., Minkema D., Van Eirdik P., Sybesma W., r978. Production characteristics of Dutch Landrace and Dutch Yorkshire pigs as related to their susceptibility for the halothaneinduced malignant hyperthermia syndrome. Livest. Prod. Sci., 5, 277-284.

Elizondo G., Addis P. B., Rempel W. E., Madero C., Martin F. B., Anderson D. B., Marple D. N., 1976. Stress response and muscle properties in Piétrain (P), Minnesota $n^{\circ}$ I (M) and $\mathrm{P} \times \mathrm{M}$ pigs. J. Anim. Sci., 43, I004-IoI4.

GERWIG C., VöGELI P., SCHWöRER D., I977. Halothane sensitivity in a positive and a negative selection line. NJF Symposium on "Muscle Function and Porcine Meat Quality ", Aug. 28th-Sept. Ist, I977, Middelfart, Danemark, I 4 p. (ronéoté).

HALL L. W., WOOLF N., BRADLEY J. W. P., JOLLY D. W., I966. Unusual reaction to suxamethonium chloride. Brit. med. J., x966, 2, 1305.

HARVEY W. R., I975. Least-squares analysis of data with unequal subclass numbers.

ARS H-4, U.S. Department of Agriculture, Beltsville, Maryland, U.S.A.

JøRGENSEN P. F., I977. Étude de la relation entre sensibilité à l'halothane, groupes sanguins et systèmes enzymatiques chez les porcs Landrace Danois (en danois). Aarsberetn. Inst. Sterelitetsforsk. I977, 20, IOI-IO8.

KING W. A., Ollivier L., BASRUR Parvathi K., 1976. Erythrocyte osmotic response test on malignant hyperthermia-susceptible pigs. Ann. Génét. Sél. anim., 8, 537-540.

LEFORT G., OLIIVIER L., SELLIER P., I975. Analyse du comportement et de la fréquence des gènes à effets visibles dans des fratries de germains et de demi-germains. Ann. Génét. Sél. anim., 7, 365-377.

McGloughlin P., Ahern C. P., McLoughiln J. V., i977. Halothane sensitivity and $\mathrm{pH}_{1}$ testing in Irish pigs. NJF Symposium on "Muscle Function and Porcine Meat Quality", Aug. 28th-Sept. Ist, I977, Middelfart, Danemark, 9 p. (ronéoté).

MiNkEMA D., EIKELENBOOM G., VAN ElDIK P., I977. Inheritance of MHS-susceptibility in pigs. In Proc. Third Int. Conf. on Production Disease in Farm Animals, Sept. I3-16, I976, 203-207, Pudoc, Wageningen, Pays-Bas.

Monin G., OlLIVIER L., SELlier P., I976. Étude du syndrome d'hyperthermie maligne chez le porc de Piétrain: premiers résultats. In Journées Rech. Porcine en France 1976, 229-238, Institut technique du Porc, Paris (Ann. Zootech., 25, 447-448, Abstr.).

Monin G., SEllier P., Olfivier L., I979. Étude de quelques caractéristiques sanguines de populations porcines françaises; relation avec le syndrome d'hyperthermie maligne. $A n n$. Rech. Vet., en préparation.

OlitVier L., LAUVERGNe J. J., 1967. Étude du déterminisme héréditaire de 1'hypertrophie musculaire du porc de Piétrain : premiers résultats. Ann. Méd. vét., 111, 104-1o9.

Olfivier L., Seiliter P., MoniN G., I975. Déterminisme génétique du syndrome d'hyperthermie maligne chez le porc de Piétrain. Ann. Génét. Sél. anim., 7, I59-I66.

OLLIVIER L., SELIIER P., MONIN G., I977. Frequency of the malignant hyperthermia syndrome (MHS) in some French pig populations: preliminary results. In Proc. Third Int. Conf. on Production Disease in Farm Animals, Sept. I3-16, 1976, 208-210, Pudoc, Wageningen, Pays-Bas.

Rasmusen B. A., Christian L. L., I976. H blood types in pigs as predictors of stress susceptibility. Science, 191, 947-948.

Riggs B. L., Judge M. D., Aberle E. D., Alitiston C. W., Wirson S. P., I974. Halothane anesthesia as a test for PSS in swine. J. Anim. Sci., 39, 162 (Abstr.).

SchwörER D., BlưM J., I977. Blasses, wässeriges Schweinefleisch : Voraussage mangelhalter Fleischbeschaffenheit bereits am lebenden Tier. Der Kleinviehzüchter, 25, 64I-650.

Smith C., BAMPTON P. R., I977. Inheritance of reaction to halothane anaesthesia in pigs. Genet. Res., Camb., 29, 287-292. 
Svbesma W., Eikelenboom G., i969. Malignant hyperthermia syndrome in pigs. Neth. J. vet. Sci., 2, I55-I60.

WeBb A. J., JoRDAN C. H. C., 1977. The halothane test in genetic improvement programmes: experiments with Piétrain-Hampshire pigs. NJF Symposium on "Muscle Function and Porcine Meat Quality ", Aug. 28th - Sept. Ist, I977, Middelfart, Danemark, 9 p. (ronéoté).

WEBB A. J., JoRDAN C. H. C., I978. Halothane sensitivity as a field test for stress-susceptibility in the pig. Anim. Prod., 26, I57-I 68.

Wilitiams C. H., LASLEY J. F., MUhrer M. E., Hedrick H. B., I975. Relationship between fulminant hyperthermia and the porcine stress syndrome. J. Anim. Sci., 41, 261 (Abstr.). 